\title{
Article \\ New Evidence of the Azimuthal Alignment of Quasars Spin Vector in the LQG U1.28, U1.27, U1.11, Cosmologically Explained
}

\author{
Reinoud Jan Slagter $1,+(\mathbb{C}$
}

1 Asfyon, Astronomisch Fysisch Onderzoek Nederland; info@asfyon.com former: University of Amsterdam, The Netherlands

* Correspondence: info@asfyon.com or reinoudjan@gmail.com; Tel.: +31643900550

+ Current address: 1405EP Bussum, The Netherlands

\begin{abstract}
There has been observational evidence about spin axes of quasars in large quasar groups correlated over hundreds of Mpc. This is seen in the radio spectrum as well as in the optical range. There is not yet a satisfactory explanation of this "spooky" alignment. This alignment cannot be explained by mutual interaction at the time that quasars manifest themselves optically. A cosmological explanation could be possible in the formation of superconducting vortices (cosmic strings) in the early universe, just after the symmetry-breaking phase of the universe. We gathered from the NASA/IPAC and SIMBAD extragalactic databases the right ascension, declination, inclination, position angle and eccentricity of the host galaxies of 3 large quasar groups to obtain the azimuthal and polar angle of the spin vectors. The alignment of the azimuthal angle of the spin vectors of quasars in their host galaxy is confirmed in the large quasar group U1.27 and compared with two other groups in the vicinity, i.e., U1.11 and U1.28. It is well possible that the azimuthal angle alignment fits the predicted azimuthal angle dependency in the theoretical model of the formation of general relativistic superconducting vortices, where the initial axially symmetry is broken just after the symmetry breaking of the scalar-gauge field.
\end{abstract}

Keywords: quasar groups; alignment spin vectors; host galaxy; cosmic strings; symmetry breaking; scalar-gauge field.

\section{Introduction}

A large quasar group (LQG) is a cluster of quasars that makes the largest astronomical structures in the current universe. Their sizes can be of the order of hundreds of Mpc. Astronomers believe that a quasar is an active galactic nuclei (AGN) with a vibrant eruption of radiation both optical and in radio range originated by a spinning (Kerr-) black hole, surrounded by an accretion disk. According to Taylor and Jagannathan[1], a LQG has an internal non-uniform distribution of spin vectors seen in the radio spectrum and the optical spectrum as observed by Hutsemekers[2]. This coherence is mysterious and cannot be explained by mutual interaction at the time scale of primordial galaxies formation but rather by use of a more advanced method. In a recent study, Slagter[3] found that the azimuthal angle of the spin vector of quasars in their host galaxies in six quasar groups, show preferred directions. This is explained through an emergent azimuthal angle dependency of the general relativistic Nielsen-Olesen (NO) vortices at the point after the symmetry breaking at grand unified theory (GUT)-scale. This review focuses on three more other LQG, studied by Clowes[4,5].

\section{Results}

From the NASA/IPAC extragalactic database and SIMBAD we extract for the three LQG U1.11, U1.27 and U.28 the right ascension, declination, inclination, position angle and eccentricity of the host galaxies. The 3-D orientation of the spin vectors can then 
be calculated ([6]). In figure 1 and 2 we plotted the azimuthal angle. Without statistical analysis one can conclude that the preferred orientations are evident. In the case of LQG U1.27, we fitted two trigonometric functions on the distribution, which can theoretically be explained (section 3).

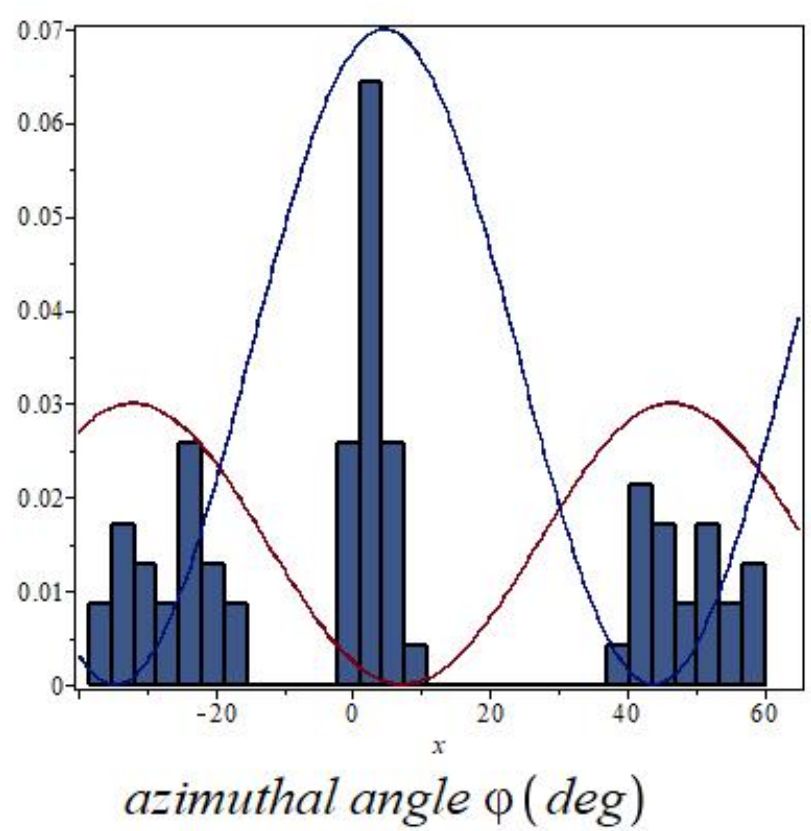

Figure 1. Plot of the azimuthal angle $\varphi$ in degrees. This shows the distribution of the azimuthal angle of the spin vectors in the LQG U1.27 (N=71) with a best-fit of two trigonometric functions with a phase shift range of $45^{\circ}$.

LQG U1.28

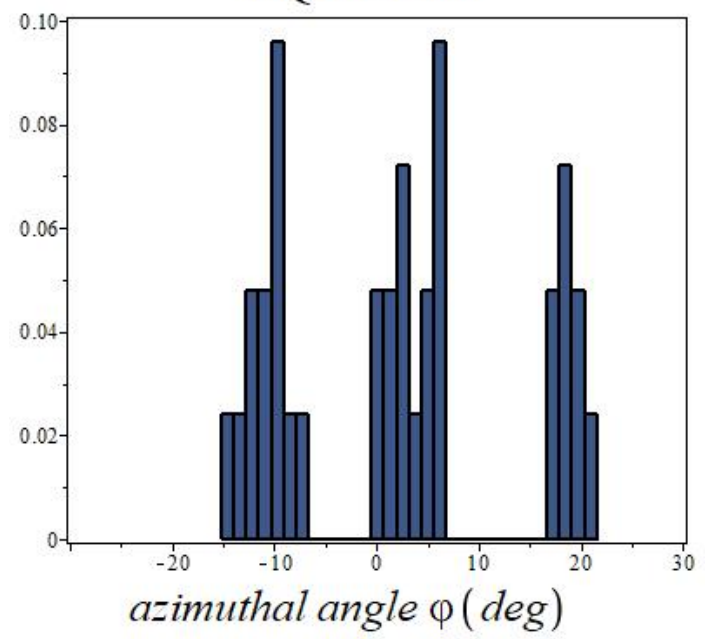

LQG U1.11

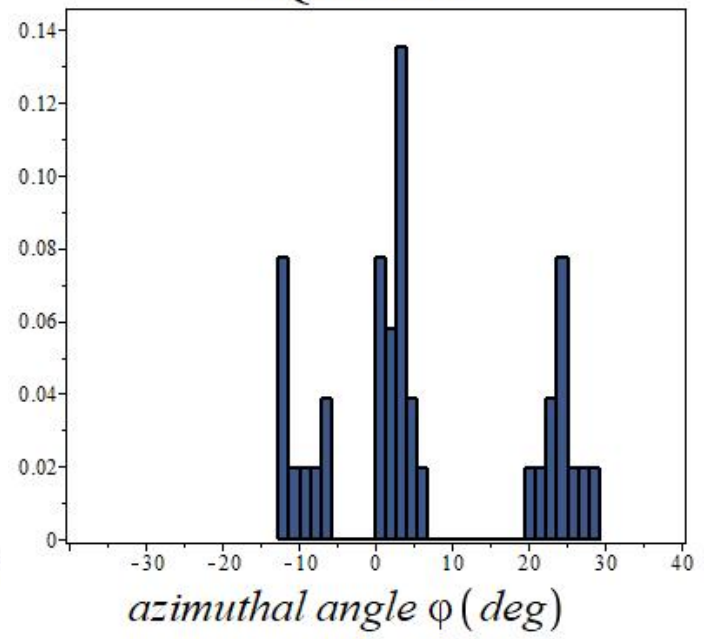

Figure 2. Plot showing the distribution of the azimuthal angle $\varphi$ for the LQG U1.28 (N=34) and U1.11 $(N=38)$.

\section{The Theoretical Model}

A linear approximation of wavelike solutions of the Einstein equations is not adequate when one is dealing with high curvature (or high energy scale), i.e., close to the horizons of black holes or in the early stage of the universe at the time of mass formation by the Higgs mechanism. There will be a "back-reaction" on the background spacetime. There is a powerful approximation method which can deal with these non linearities: 
the multiple-scale method. Pioneer work was done by [7]. One expands the relevant fields ([8])

$$
V_{i}=\sum_{n=0}^{\infty} \frac{1}{\omega^{n}} F_{i}^{(n)}(\mathbf{x}, \xi)
$$

where $\omega$ represents a dimensionless parameter ("frequency"), which will be large. Further, $\xi=\omega \Theta(\mathbf{x})$, with $\Theta$ a scalar (phase) function on the manifold. The small parameter $\frac{1}{\omega}$ can also be the ratio of the characteristic wavelength of the perturbation to the characteristic dimension of the background. On warped spacetimes it could also be the ratio of the extra dimension to the background dimension. In the vacuum case, we expand the metric

$$
g_{\mu v}=\bar{g}_{\mu v}+\frac{1}{\omega} h_{\mu v}(\mathbf{x}, \xi)+\frac{1}{\omega^{2}} k_{\mu v}(\mathbf{x}, \xi)+\ldots
$$

where we defined

$$
\frac{d g_{\mu v}}{d x^{\sigma}}=g_{\mu v, \sigma}+\omega l_{\sigma} \dot{g}_{\mu v}, \quad g_{\mu v, \sigma}=\frac{\partial g_{\mu v}}{\partial x^{\sigma}}, \quad \dot{g}_{\mu v}=\frac{\partial g_{\mu v}}{\partial \xi},
$$

with $l_{\mu}=\frac{\partial \Theta}{x^{\mu}}$. One then says that

$$
V_{i}=\sum_{n=-m}^{\infty} \frac{1}{\omega^{n}} F_{i}^{(n)}(\mathbf{x}, \xi)
$$

is an approximate wavelike solution of order $\mathrm{n}$ of the field equation, if $F_{i}^{(n)}=0, \forall n$. One can substitute the expansion into the field equations. The Ricci tensor then expands as

$$
R_{\mu v} \rightarrow \omega R_{\mu v}^{(-1)}+\left(\bar{R}_{\mu v}+R_{\mu \nu}^{(0)}\right)+\frac{1}{\omega} R_{\mu \nu}^{(1)}+\ldots
$$

By equating the subsequent orders to zero, we obtain

$$
\begin{gathered}
R_{\mu \nu}^{(-1)}=0=\frac{1}{2} \bar{g}^{\beta \lambda}\left(l_{\lambda} l_{\mu} \ddot{h}_{\beta v}+l_{\nu} l_{\beta} \ddot{h}_{\mu \lambda}-l_{\lambda} l_{\beta} \ddot{h}_{\mu v}-l_{\nu} l_{\mu} \ddot{h}_{\beta \lambda}\right), \\
R_{\mu \nu}^{(0)}+\bar{R}_{\mu v}=0, \quad R_{\mu \nu}^{(1)}=0, \quad \ldots .
\end{gathered}
$$

Here we used $l_{\mu} l^{\mu}=0$. The rapid variation is observed in the direction of $l_{\mu}$. In the radiative outgoing Eddington-Finkelstein coordinates, we have $x^{1}=u=\Theta(\mathbf{x})=t-r$ and $l_{\mu}=(1,0,0,0)$ while the bar stands for the background.

\subsection{Formation of vortices}

In a recent study of [9-11] we applied this non-linear approximation scheme on a FLRW spacetime. We considered the matter contribution of a gauged complex scalar (Higgs) field. Physicists are now convinced that this field plays a fundamental role in the early universe and is responsible for the symmetry breaking in the Standard Model of particle physics. The experimental verification came by the recently observed Higgs particle at CERN. The same field has lived up to its reputation in superconductivity, where the field act as an order parameter to describe the formation of Cooper pairs. The scalar field is combined with a gauge field, parameterized as $\Phi=\eta X(\mathbf{x}) e^{i n \varphi}$ and $A_{\mu}(\mathbf{x})=\frac{n}{e}(P(\mathbf{x})-1) \nabla_{\mu} \varphi$, with $n$ the topological charge or winding number. The trapped flux of the vortex is expressed as $n \frac{2 \pi h}{e}$. The formation of a lattice of quantized magnetic flux tubes was first observed by [12] and are described by the famous equations of [13]. In these models, one needs the quartic potential of the Higgs field, i.e., $V(\Phi)=$ $\frac{1}{8} \lambda\left(\Phi \Phi^{*}-\eta^{2}\right)^{2}$, with $\eta$ the vacuum expectation value. Further, $\frac{m_{\Phi}}{m_{A}}=\frac{e^{2}}{\lambda}$ is the ratio of the scalar to gauge masses. This potential leads to a nonzero $\eta$ and spontaneous 
breaking of the $\mathrm{U}(1)$ symmetry (the parameters are in general temperature dependent). The forces existing between the vortices are the electromagnetic and scalar force. When the vortices get close together, the problem becomes non-linear and the resulting force depends on the ratio $\frac{e^{2}}{\lambda}$. For details see, for example, the text books of Felsager [14] and Weinberg [15]. Moreover, when the vortices are formed in the early stage of the universe, then gravity will come into play. These general relativistic vortex solutions are known as "cosmic strings" ([16]; [17]) when they extend to cosmological dimensions. They could possibly explain the observed void and filament structures in the universe.

We expand the scalar and gauge field to second order as

$$
\begin{aligned}
A_{\mu} & =\bar{A}_{\mu}(\mathbf{x})+\frac{1}{\omega} B_{\mu}(\mathbf{x}, \xi)+\frac{1}{\omega^{2}} C_{\mu}(\mathbf{x}, \xi)+\ldots, \\
\Phi & =\bar{\Phi}(\mathbf{x})+\frac{1}{\omega} \Psi(\mathbf{x}, \xi)+\frac{1}{\omega^{2}} \Xi(\mathbf{x}, \xi)+\ldots,
\end{aligned}
$$

where we write the subsequent orders of the scalar field as

$$
\bar{\Phi}=\eta \bar{X}(t, r) e^{i n_{1} \varphi}, \quad \Psi=Y(t, r, \xi) e^{i n_{2} \varphi}, \quad \Xi=Z(t, r, \xi) e^{i n_{3} \varphi},
$$

with $n_{i}$ the subsequent winding numbers.

\subsection{The azimuthal angle dependency: breaking the axial symmetry}

The azimuthal angle $\varphi$ does not reach the partial differential equations (PDE) in the unperturbed case. By quantum fluctuations, the vortex excite in higher $n$-state and will dissociate into $n$ well separated $n=1$ vortices $^{1}$, because the energy of the configuration is proportional with $n^{2}$. The topological characterization is a set of isolated points with winding numbers $n_{i}$ (the zero's of $\Phi$ ), with $n=n_{1}, n_{2}, \ldots$. This n-vortices solution represents a finite energy configuration. However, an imprint will be left over of the azimuthal dependency of the orientation of the clustering of Abrikosov vortices lattice in the general relativistic situation. So the axial symmetry is dynamically broken. The azimuthal dependency emerge already to first order in the approximation. For example, the energy-momentum tensor $\bar{T}_{t \varphi}=0$, while the first order perturbation becomes

$$
T_{t \varphi}^{(0)}=\bar{X} \bar{P} \dot{Y} n_{1} \sin \left(n_{2}-n_{1}\right) \varphi
$$

However, in $T_{t \varphi}^{(1)}$ there appears terms like $\cos \left(n_{2}-n_{1}\right) \varphi$ and $\sin \left(n_{3}-n_{1}\right) \varphi$. The perturbative appearance of a non-zero energy-momentum component $T_{t \varphi}$ can be compared with the phenomenon of bifurcation along the Maclaurin-Jacobi sequence of equilibrium ellipsoids of self-gravitating compact objects, signalling the onset of secular instabilities ([18]). This shows a similarity with the Goldstone-boson modes of spontaneously broken symmetries of continuous groups. The recovery of the $\mathrm{SO}(2)$ symmetry from the equatorial eccentricity takes place at a time comparable to the emission of gravitational waves.

The particular orientation of the ellipsoid in the frame $(r, \varphi, z)$ expressed as $\varphi_{0} \equiv$ $\varphi\left(t_{0}\right)$, is at $t>t_{0}$ and determined by the transformation $\varphi \rightarrow \varphi_{0}-J t$, where $J$ is the rotation frequency (circulation or "angular momentum") of the coordinate system. The angle $\varphi_{0}$ is fixed arbitrarily at the onset of symmetry breaking.

\subsection{The pure gravitational radiation case}

So far, we found that temporarily off-diagonal terms occurred in the perturbative approach of the Einstein scalar gauge field. What remains unclear is if the breaking

1 The stability of the configuration depends on parameter $\lambda([15])$ 
of the axially symmetry already appears in the vacuum case like in the vicinity of a black hole spacetime. It is conjectured that the formation of primordial (Kerr-) black holes (and so quasars) happened in the early stages of the evolution of the universe before the stars were formed. Therefore, consider the radiative Vaidya spacetime in Eddington-Finkelstein coordinates ${ }^{2}$

$$
d s^{2}=-\left(1-\frac{2 M(u)}{r}\right) d u^{2}-2 d u d r+r^{2}\left(d \theta^{2}+\sin ^{2} \theta d \varphi^{2}\right),
$$

which is the Schwarzschild black hole spacetime with $u=t-r-2 M \log \left(\frac{r}{2 M}-1\right)$. Here we used $l_{\mu} l^{\mu}=0$. In the radiative coordinates, we have $x^{1}=u=\Theta(\mathbf{x})$ and $l_{\mu}=(1,0,0,0)$. From Eq.(6) we obtain

$$
h_{r r}=h_{r \theta}=h_{r \varphi}=0, \quad h_{\varphi \varphi}=-\sin ^{2} \theta h_{\theta \theta}
$$

From the zero-order equations Eq.(7) we obtain

$$
\ddot{k}_{r r}=0, \quad \dot{h}_{\theta \theta}=r \partial_{r} \dot{h}_{\theta \theta}, \quad \dot{h}_{\theta \varphi}=r \partial_{r} \dot{h}_{\theta \varphi} .
$$

So one writes

$$
h_{\theta \theta}=r \alpha(u, \theta, \varphi, \xi), \quad h_{\theta \varphi}=r \beta(u, \theta, \varphi, \xi), \quad h_{\varphi \varphi}=-r \alpha \sin ^{2} \theta
$$

Further, we have

$$
\begin{gathered}
\ddot{k}_{r \theta}=\frac{1}{r}\left(2 \dot{\alpha} \cot \theta+\partial_{\theta} \dot{\alpha}+\frac{1}{\sin ^{2} \theta} \partial_{\varphi} \dot{\beta}\right), \\
\ddot{k}_{r \varphi}=\frac{1}{r}\left(\dot{\beta} \cot \theta-\partial_{\varphi} \dot{\alpha}+\partial_{\theta} \dot{\beta}\right), \\
\frac{d M}{d u}=-\frac{\ddot{k}_{\phi \phi}+\sin ^{2} \theta \ddot{k}_{\theta \theta}}{4 \sin ^{2} \theta}-\frac{1}{2} r \dot{h}_{u u}-\frac{1}{4}\left(\dot{\alpha}^{2}+\frac{\dot{\beta}^{2}}{\sin ^{2} \theta}\right)+\frac{1}{4}\left(\ddot{\alpha}^{2}+\frac{\ddot{\beta}^{2}}{\sin ^{2} \theta}\right) .
\end{gathered}
$$

Not all the components of $h_{\mu \nu}$ and $k_{\mu \nu}$ are physical, so one needs some extra gauge conditions. Suitable choice of $\alpha$ and $\beta$ (Choquet-Bruhat uses, for example, $\alpha=0, \beta=$ $g(u) h(\xi) \sin \theta)$, leads to a solution to second order which is in general not axially symmetric. We can integrate these zero order equations with respect to $\xi$. One obtains then some conditions on the background fields, because terms like $\int \dot{\alpha} d \xi$ disappear. From Eq.(18), we obtain

$$
\frac{d M}{d u}=-\frac{1}{4 \tau} \int_{0}^{\tau}\left(\dot{\alpha}^{2}+\frac{\dot{\beta}^{2}}{\sin ^{2} \theta}\right) d \xi,
$$

which is the back-reaction of the high-frequency disturbances on the mass $M . \tau$ is the period of $\dot{h}_{\mu v}$. This expression can be substituted back into Eq.(18). However, in the non-vacuum case, the right-hand side will also contain contributions from the matter fields. In order to obtain propagation equations for $h_{\mu \nu}$ and $k_{\mu \nu}$, one proceeds with the next order equation $R_{\mu \nu}^{(1)}=0$. First of all, Eq.(16), (17) are consistent with $R_{r \varphi}^{(1)}=0$ and $R_{r \theta}^{(1)}=0$. Further, one obtains propagation equations for $\alpha$ and $\beta$ and for some second order perturbations, such as $k_{\varphi \varphi}$. Moreover, the $(\varphi, \theta)$-dependent part of the PDE's for $\alpha$ and $\beta$ (say $A(\theta, \varphi), B(\theta, \varphi)$ ) can be separated (for the case $k_{\theta \varphi} \neq 0$ ):

$$
\partial_{\varphi} B+2 \sin \theta \cos \theta A+\sin ^{2} \theta \partial_{\theta} A=0,
$$

\footnotetext{
2 This spacetime is also applied to describe the evaporation of a black hole by hawking radiation in a quantum mechanical way
} 


$$
\sin ^{2} \theta \partial_{\theta \theta} A+7 \sin \theta \cos \theta \partial_{\theta} A+4 \cot \theta \partial_{\varphi} B+2\left(5 \cos ^{2} \theta-1\right) A+2 \partial_{\theta \varphi} B=0 .
$$

A non-trivial simple solution is

$$
A=\frac{\cos \theta(\sin \varphi+\cos \varphi)}{\sin ^{3} \theta}, \quad B=\frac{\sin \varphi-\cos \varphi}{\sin ^{2} \theta}+G(\theta),
$$

with $G(\theta)$ arbitrary. So the breaking of the spherically and axially symmetry is manifest.

\section{Conclusions}

There is clear new observational evidence for the azimuthal alignment of the spin vectors of quasars in three new studied LQG. This research presents a new argument about the theoretical explanation of the axial symmetry breaking in a non-linear perturbation scheme considering a vacuum black hole spacetime in radiative coordinates. The recently discovered 13 billion years old quasar P172+18 powered by a supermassive black hole, is all the more reason to believe that the formation of these objects took place in the very early universe.

\section{References}

1. Taylor, A.R.; Jagannathan, P. Alignments of radio galaxies in deep radio imaging of ELAIS N1. Mon. Not. R. Astron. Soc., 459, L36 2016.

2. Hutsemekers, D., e.a. Alignment of quasar polarizations with large-scale structures. AEA, 572, A18 2014.

3. Slagter, R.J.; Miedema, P.G. On the azimuthal alignment of quasars spin vector in large quasar groups and cosmic strings. Mon. Not. Roy. Astron. Soc., 501, 30542021.

4. Clowes, R.G.e.a. Two close large quasar groups of size $=350 \mathrm{Mpc}$ at z =1.2. Mon. Not. R. Astron. Soc., 419, 5562012.

5. Clowes, R.G.e.a. A structure in the early universe at $\mathrm{z}=1.3$ that exceeds the homogeneity scale of the R-W concordance cosmology. Mon. Not. R. Astron. Soc., 429, 29102013.

6. Pajowska, P., e.a. Investigation of the orientation of galaxies in clusters: the importance, methods and results of research. J. Cosm. Astroparticles, 02, 0052019.

7. Choquet-Bruhat, Y. Construction de solutions radiatives approchees des equations d'Einstein. Commun. math. Phys. , 12, 16 1968.

8. Slagter, R.J. High-Frequency Perturbations and Gravitational Collapse in Gravity Theory Coupled with a Higgs Field. Astrophys. J. 1986, 307, 20. doi:10.1086/164388.

9. Slagter, R.J. Alignment of Quasar Polarizations on Large Scales Explained by Warped Cosmic Strings. Journal of Modern Physics 2016, 08, 163-180. doi:10.4236/jmp.2017.82015.

10. Slagter, R.J. Alignment of Quasar Polarizations on Large Scales Explained by Warped Cosmic Strings PART II: The Second Order Contribution. Journal of Modern Physics 2017, 08, 163-180. doi:10.4236/jmp.2017.82015.

11. Slagter, R.J. Evidence of cosmic strings by the observation of the alignment of quasar polarization axes on Mpc scale. Int. J. Mod. Phys. D 2018, 27, 1850094. doi:10.1142/s0218271818500943.

12. Abrikosov, A.A. On the Magnetic Properties of Superconductors of the Second Group. Sov. Phys. JETP, 5, 11741957.

13. Ginzberg, V.L.; Landau, L.D. On the Theory of Superconductivity. Zh.Eksp.Teor.Fiz., 20,1064 1950.

14. Felsager, B. Geometry, Particles and Fields; Odense Univ. Press, Odense, 1998.

15. Weinberg, E.J. Classical Solutions in Quantum Field Theory; Cambridge Univ. Press, Cambridge, 2012.

16. Garfinkle, D. General Relativistic Strings. Phys. Rev. D. 32, 13231985.

17. Vilenkin, A.; Shellard, E.P.S. Cosmic Strings and Other Topological Defects; Cambridge Univ. Press, Cambridge, 1994.

18. Gondek-Rosinska, D.; Gourgoulhon, E. Jacobi-like bar mode instability of relativistic rotating bodies. Phys. Rev. D66, 044021 2002. 